\section{Clinical relevance of quantified fundus autofluorescence in diabetic macular oedema}

S Yoshitake, T Murakami, A Uji, N Unoki, Y Dodo, T Horii and $\mathrm{N}$ Yoshimura
Department of

Ophthalmology and Visual Sciences, Kyoto University Graduate School of Medicine, Kyoto, Japan

Correspondence:

T Murakami, Department of Ophthalmology and Visual Sciences, Kyoto University Graduate School of Medicine, 54 ShogoinKawaracho, Sakyo, Kyoto 606-8507, Japan

Tel: +81 75751 3250;

Fax: +81757520933.

E-mail: mutomo@kuhp.

kyoto-u.ac.jp

Received: 27 September 2014

Accepted in revised form: 28 January 2015

Published online:

13 March 2015

\begin{abstract}
Purpose To quantify the signal intensity of fundus autofluorescence (FAF) and evaluate its association with visual function and optical coherence tomography (OCT) findings in diabetic macular oedema (DMO).

Methods We reviewed 103 eyes of 78 patients with DMO and 30 eyes of 22 patients without DMO. FAF images were acquired using Heidelberg Retina Angiograph 2, and the signal levels of FAF in the individual subfields of the Early Treatment Diabetic Retinopathy Study grid were measured. We evaluated the association between quantified FAF and the logMAR VA and OCT findings. Results One hundred and three eyes with DMO had lower FAF signal intensity levels in the parafoveal subfields compared with 30 eyes without DMO. The autofluorescence intensity in the parafoveal subfields was associated negatively with $\log$ MAR VA and the retinal thickness in the corresponding subfields. The autofluorescence levels in the parafoveal subfield, except the nasal subfield, were lower in eyes with autofluorescent cystoid spaces in the corresponding subfield than in those without autofluorescent cystoid spaces. The autofluorescence level in the central subfield was related to foveal cystoid spaces but not logMAR VA or retinal thickness in the corresponding area. Conclusions Quantified FAF in the parafovea has diagnostic significance and is clinically relevant in DMO.

Eye (2015) 29, 662-669; doi:10.1038/eye.2015.25; published online 13 March 2015
\end{abstract}

\section{Introduction}

Diabetic retinopathy (DR), a major cause of visual impairment in patients of working age, is mainly mediated via angiogenic complications and diabetic macular oedema (DMO). ${ }^{1}$ Diabetes mellitus leads to disruption of the blood-retinal barrier, and the accumulated blood components in the extravascular spaces exacerbate macular thickening and functional disturbance., ${ }^{2,3}$ Although the recent application of antivascular endothelial growth factor therapy has improved the visual prognosis in patients with $\mathrm{DMO}_{,}^{4,5}$ a method to objectively evaluate the clinical findings and the pathogenesis remains to be developed.

Fluorescein angiography, an invasive modality, provides qualitative and quantitative assessments of the vascular hyperpermeability in DMO. Clinical application of optical coherence tomography (OCT) enables measurement of the retinal thickness in healthy and diabetic eyes. Clinicians recently began observing the fine pathological structures of intraretinal lesions on spectral-domain (SD) OCT images with higher resolution and reduced speckle noises. ${ }^{6}$ Automated quantification of the retinal thickness especially is the gold standard for objectively evaluating the severity of DMO; many publications have reported a modest correlation between the macular thickness and visual impairment in eyes with DMO. ${ }^{7}$ Despite the clinical relevance of OCT measurements, errors of segmentation of the inner limiting membrane or retinal pigment epithelium (RPE) sometimes occur, leading to incorrect measurements of the mean retinal thickness.

Fundus autofluorescence (FAF) is another modality for evaluating chorioretinal diseases. The autofluorescence signals are widely thought to be emitted mainly from lipofuscin in the RPE cells, which is derived from the debris of incompletely digested photoreceptor outer segments and, to a lesser extent, from retinol or related proteins in the photoreceptors. ${ }^{8}$ Increase and decrease in FAF signal levels have been reported in pathological states. Hypoautofluorescence often corresponds to decreased fluorophores in diseases at the 
photoreceptor-RPE interface in geographic atrophy or retinitis pigmentosa. ${ }^{9,10}$ Macular pigment blocks the autofluorescence signals from the RPE, and opsins (rhodopsin or cone opsins) also block 488-nm excitation light in healthy eyes, whereas well-demarcated hyperautofluorescence is delineated in areas corresponding to foveal cystoid spaces in DMO and retinal vein occlusion. ${ }^{11-13} \mathrm{~A}$ recent publication found that the areas of autofluorescent cystoid spaces have clinical relevance in eyes treated with bevacizumab. ${ }^{14}$ FAF also might have potential use in observing RPE changes after retinal photocoagulation. ${ }^{15}$ In addition, it is speculated that oedematous changes in retinal parenchyma block the autofluorescence and concomitantly decrease the FAF signal levels in macular oedema due to retinal vascular diseases. However, it is difficult to measure the autofluorescence intensity, because the signal levels are modulated by the cornea and crystalline lens in patients with diabetes. ${ }^{16,17}$

We investigated a novel method to quantify the relative levels of FAF intensity in individual subfields of the Early Treatment Diabetic Retinopathy Study (ETDRS) grid in DMO and evaluated the clinical relevance compared with OCT findings.

\section{Materials and methods}

\section{Patients}

We retrospectively reviewed 103 eyes of 78 patients (mean, 63.5 \pm 9.9 years; range, 33-84) who visited the Department of Ophthalmology of Kyoto University Hospital from June 2010 to June 2013. Two eyes had mild nonproliferative diabetic retinopathy (NPDR), 64 eyes had moderate NPDR, 19 eyes had severe NPDR, and 18 eyes had proliferative diabetic retinopathy (PDR). The inclusion criteria were the presence of centreinvolved DMO based on OCT measurements and the availability of SD-OCT and FAF images of sufficient quality obtained on the same day. We excluded eight eyes in which severe intraretinal lesions containing microaneurysms, retinal haemorrhages, and hard exudates blocked the autofluorescence signals from the $\mathrm{RPE}$ in any subfield of the inner ring $(1-3 \mathrm{~mm})$ or the central subfield of the ETDRS grid. Other exclusion criteria were the presence of any other chorioretinal diseases, including age-related maculopathy and agerelated macular degeneration, severe media opacity, a history of treatment of DMO, cataract surgery within 3 months, or any major surgery other than cataract extraction within 1 year. We also evaluated 30 eyes of 22 patients with DR but without either clinically significant macular oedema or centre-involved DMO that were matched according to age and DR severity
(19 eyes with moderate NPDR, 5 eyes with severe NPDR, and 6 eyes with PDR) that served as a control group. Six eyes with DMO or one eye without DMO was pseudophakic. All research and measurements adhered to the tenets of the Declaration of Helsinki. The ethics committee of Kyoto University Graduate School of Medicine approved the study protocol. Informed consent was obtained from the participants after the full explanation of the nature and possible consequences of the study.

\section{Optical coherence tomography}

After comprehensive ophthalmological examinations, including measurement of the best-corrected visual acuity (VA), slit-lamp biomicroscopy, and colour fundus photography, sectional images of the macula were scanned using SD-OCT (Spectralis OCT, Heidelberg Engineering, Heidelberg, Germany) followed by evaluation of qualitative and quantitative OCT parameters. We measured the mean retinal thickness of the central subfield and the four quadrants (superior, nasal, inferior, and temporal) of the inner ring (1-3 mm) of the ETDRS grid using a two-dimensional OCT map constructed by raster scans, as described previously. ${ }^{18}$ We further assessed the qualitative OCT parameters: the presence of foveal cystoid spaces and foveal serous retinal detachment (SRD), and the status of the external limiting membrane (ELM) at the fovea. ${ }^{18-20}$ These parameters were applied to further analyses and compared with the FAF signal intensity.

\section{Fundus autofluorescence}

FAF images of the macula were acquired using a scanning laser ophthalmoscope (Heidelberg Retina Angiograph 2, Heidelberg Engineering). Autofluorescence signals through a $500-n m$ filter with excitation by 488 -nm laser light were detected in an area $30 \times 30$ degrees centred on the fovea. The gain level was adjusted to delineate the major vessels and the disc on a single scan image, followed by averaging for sufficient quality.

We then quantified the relative autofluorescence levels in individual quadrants of the inner ring and the central subfield of the ETDRS grid on the FAF images (Supplementary Figure). We measured and averaged the autofluorescence signal levels as 'mean autofluorescence intensity' in individual subfields using the Image J software (National Institutes of Health, Bethesda, MD, USA). We quantified the mean signal intensity of 1000 pixels in the optic disc where the RPE and photoreceptors are absent as the zero point in individual images. A value of 1 was assigned to the signal intensity in areas outside the ETDRS grid $(\sim 6 \mathrm{~mm})$, because the pathological 
hyperautofluorescence or hypoautofluorescence was delineated mainly within the ETDRS grid $(\sim 6 \mathrm{~mm})$ and the signal levels outside the ETDRS grid were decreased by intraretinal lesions and retinal vasculature alone. In this way, we calculated the mean signal levels in 1000 pixels with the highest signals in the individual quadrants (superior, nasal, inferior, and temporal) outside the grid using the Image J software, followed by averaging of these subfields (referred to as the autofluorescence intensity in the areas outside the ETDRS grid). We calculated the relative autofluorescence intensity according to the formula:

Relative autofluorescence intensity in each subfield autofluo (mean intensity in each subfield) - autofluo (optic disc) autofluo(areas outside the ETDRS grid) - autofluo (optic disc)

where autofluo indicates autofluorescence intensity.

We evaluated the agreement of the values between two independent graders (intraclass correlation coefficient, 0.993), and the average was applied to further analysis.

We further evaluated the increased FAF levels in the areas corresponding to the cystoid spaces and referred to them as autofluorescent cystoid spaces in this study. Macular pigments are present mainly in the outer plexiform layer (OPL) in the macula and block the autofluorescence signals from the RPE in healthy eyes. Well-demarcated oval or round areas with higher levels of FAF signals were delineated around the macula in eyes with cystoid spaces on OCT images. ${ }^{11-13}$ We then assessed the presence of the autofluorescent cystoid spaces in individual subfields of the ETDRS grid.

\section{Statistical analysis}

The results are expressed as the mean \pm SD. Student's $t$-test was used to compare the quantitative data populations with normal distributions and equal variance. The data were analysed using the MannWhitney $U$ test for populations with non-normal distributions or unequal variance. Univariate linear regression analysis was performed to test the statistical correlation. $P<0.05$ was considered significant.

\section{Results}

\section{Clinical relevance of autofluorescence signal levels in parafoveal subfields}

We quantified the mean signal intensity of the FAF in the central 1-mm subfield and individual quadrants of the parafovea in eyes with centre-involved DMO and showed lower signal levels in the central subfield than in the parafoveal subfield in the control eyes (Table 1). Eyes with DMO had lower levels of FAF signal intensity in the parafoveal subfields than those without DMO, compared
Table 1 Mean autofluorescence intensity in individual subfields in eyes with and without $\mathrm{DMO}$

\begin{tabular}{lccr}
\hline Subfield & $\begin{array}{c}\text { With DMO } \\
(\mathrm{n}=103)\end{array}$ & $\begin{array}{c}\text { Without DMO } \\
(\mathrm{n}=30)\end{array}$ & $\begin{array}{r}\text { P-value } \\
\hline \text { Central }\end{array}$ \\
Superior & $0.198 \pm 0.090$ & $0.177 \pm 0.100$ & 0.194 \\
Nasal & $0.303 \pm 0.112$ & $0.388 \pm 0.095$ & $<0.001$ \\
Inferior & $0.337 \pm 0.115$ & $0.420 \pm 0.121$ & 0.002 \\
Temporal & $0.317 \pm 0.124$ & $0.435 \pm 0.122$ & $<0.001$ \\
\hline
\end{tabular}

Abbreviation: DMO, diabetic macular oedema.

with no differences in the central subfield (Table 1, Figure 1). The autofluorescence intensity in the parafoveal subfields was also negatively correlated with the logarithm of minimum angle of resolution (logMAR) VA compared with that in the central subfield in eyes with $\mathrm{DMO}$, and the correlation was the most significant in the nasal subfield (Figure 2).

\section{Relationship between FAF signal intensity and OCT findings}

Recent publications have reported that hyperautofluorescence was delineated in the areas corresponding to cystoid spaces, which prompted us to investigate the relationship between autofluorescent cystoid spaces and the autofluorescence intensity. ${ }^{11-13}$ Ninety-five eyes (92.2\%) with autofluorescent cystoid spaces in the central subfield had higher signal intensities of FAF than eight eyes without them (Supplementary Table S1). The incidence of autofluorescent cystoid spaces in the parafoveal subfields was lower than that in the central subfield. Eyes with autofluorescent cystoid spaces in the parafoveal subfields, except the nasal subfield, had lower signal intensities in the corresponding subfields than those without autofluorescent cystoid spaces (Supplementary Table S1). We showed that the mean retinal thicknesses were correlated negatively with the FAF signal intensities in individual parafoveal subfields, although there was no association between the central subfield (CSF) thickness and the central autofluorescence intensity (Table 2, Figure 3).

We evaluated the relationship between the signal intensity of the FAF and the quantitative or qualitative OCT parameters at the fovea and found that the CSF thickness was associated negatively with the mean autofluorescence intensity in the parafoveal subfield but not with that in the central subfield (Supplementary Table S2). Among three foveal pathomorphologies, either foveal cystoid spaces or SRDs often contribute to macular thickening, which prompted us to investigate the relationship between the mean autofluorescence intensity and these OCT findings. Eyes with foveal cystoid spaces on OCT images had higher FAF signals in the central 
a

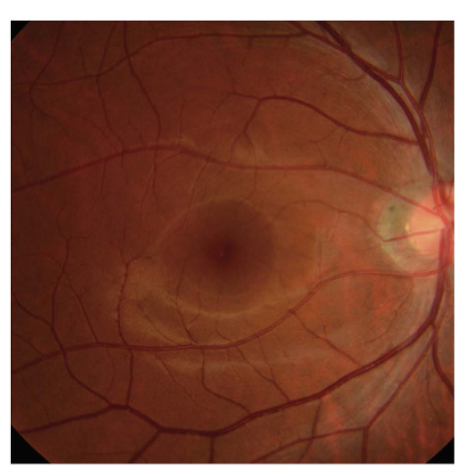

d

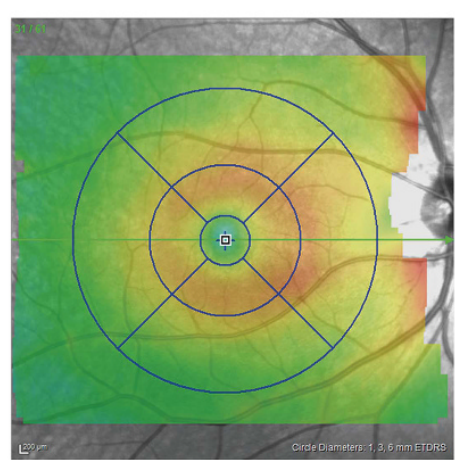

g

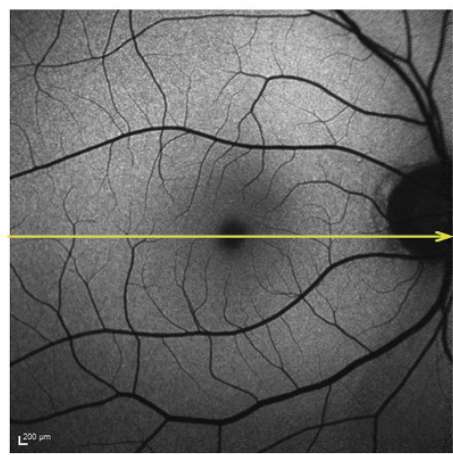

j

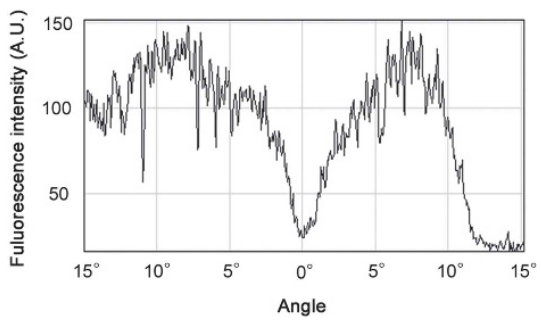

b

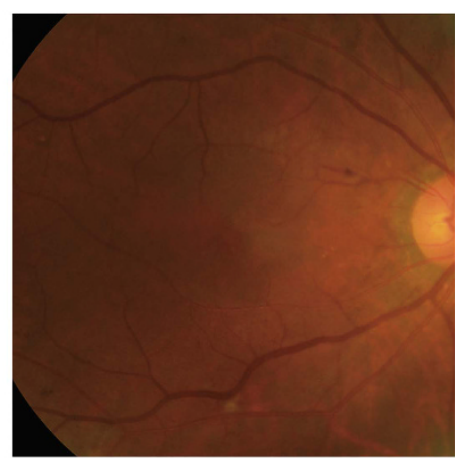

e

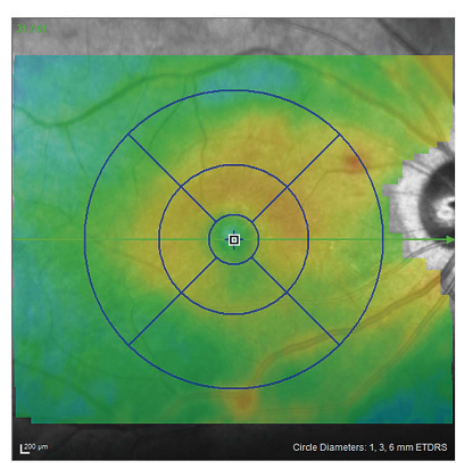

h

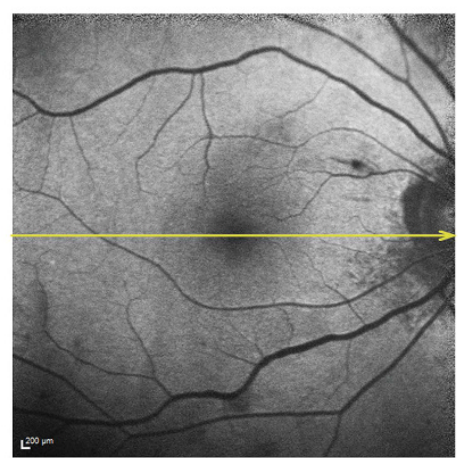

k

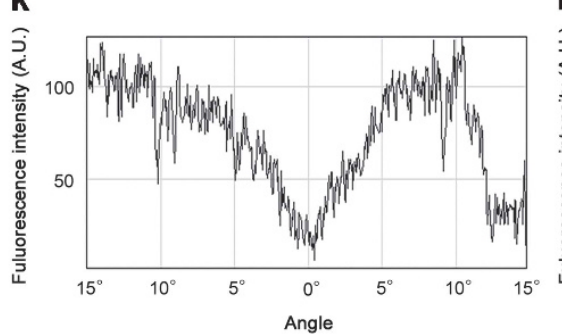

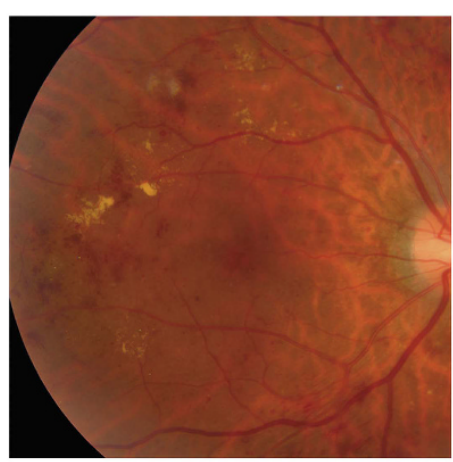

f

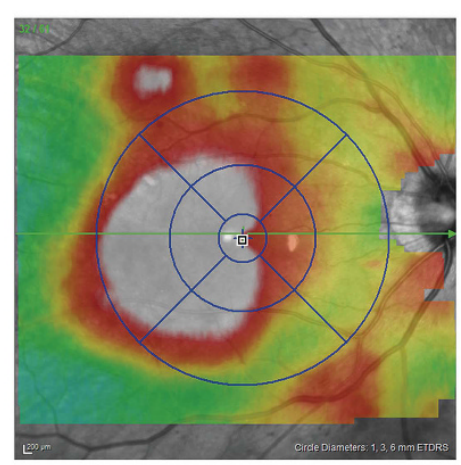

i
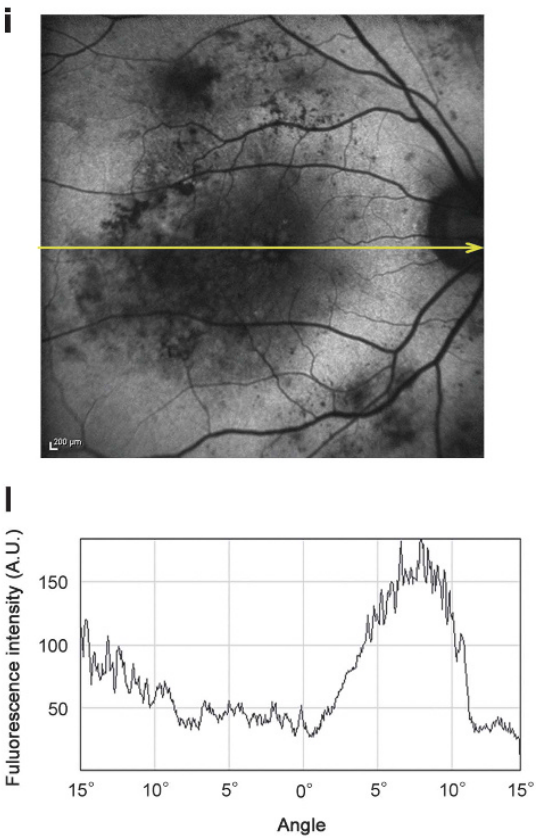

Figure 1 Representative cases. (a, d, g, j) A healthy eye , (b, e, h, k) an eye with DR but not with clinically significant macular oedema or centre-involved DMO, and ( $, f, i, 1)$ an eye with centre-involved DMO . A two-dimensional OCT map shows the presence (f) or absence $(d, e)$ of macular thickening compared with colour fundus photographs $(a-c)$. An FAF image in an eye with DR but not with DMO has a sharp trough of signal intensity at the fovea $(h, k)$ similar to that in a healthy eye $(g, j)$. In comparison, a larger area with decreased FAF signals is seen in an eye with centre-involved DMO (i, l). (j-1) Signal levels dissecting the fovea (yellow arrows) in FAF images (third row) are shown.

subfield than those without foveal cystoid spaces; there were no differences in the FAF signal levels in the parafoveal subfields (Supplementary Table S3). We did not find significant differences in the autofluorescence levels in any subfields between eyes with and without foveal SRD (Supplementary Table S4). Finally, eyes with 

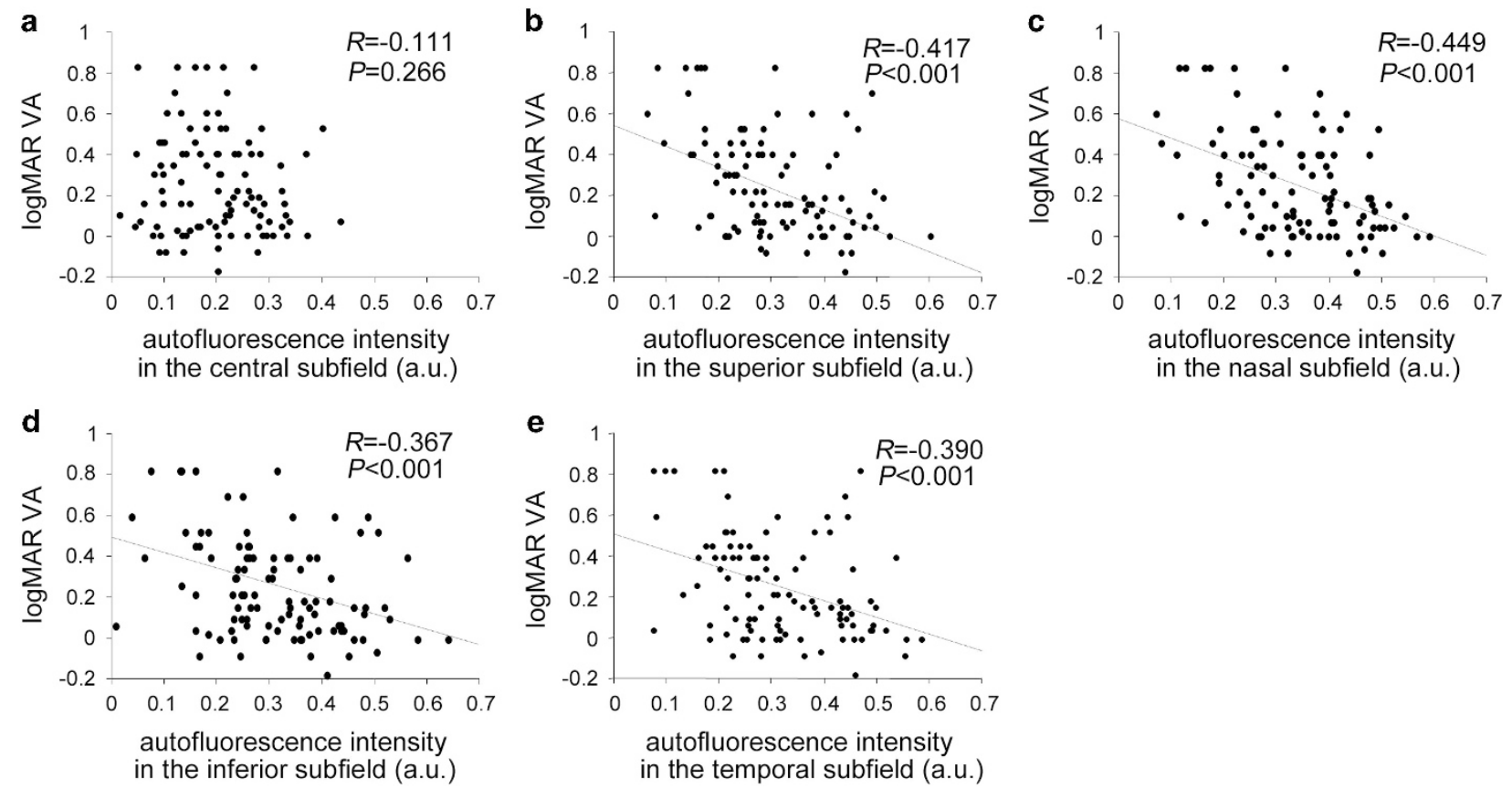

Figure 2 Association of the logMAR VA with the mean signal intensity of FAF in the (a) central, (b) superior, (c) nasal, (d) inferior, and (e) temporal subfields of ETDRS grid in DMO.

Table 2 Relationship between mean retinal thickness and FAF signal intensity of individual subfields in diabetic macular oedema

\begin{tabular}{lrr}
\hline Subfield & $\begin{array}{c}\text { Mean retinal } \\
\text { thickness }(\mu \mathrm{m})\end{array}$ & $\begin{array}{c}\text { Association with } \\
\text { intensity of } F A F\end{array}$ \\
\hline Central & $471.5 \pm 138.1$ & $R=-0.066, P=0.505$ \\
Superior & $444.7 \pm 107.3$ & $R=-0.512, P<0.001$ \\
Nasal & $424.8 \pm 93.8$ & $R=-0.430, P<0.001$ \\
Inferior & $437.0 \pm 96.6$ & $R=-0.493, P<0.001$ \\
Temporal & $459.0 \pm 119.4$ & $R=-0.469, P<0.001$ \\
\hline
\end{tabular}

Abbreviation: FAF, fundus autofluorescence.

disrupted ELM had lower FAF signals in the superior and temporal subfields than in those with intact ELM (Supplementary Table S5).

\section{Discussion}

Several publications have qualitatively assessed hyperautofluorescence in areas corresponding to foveal cystoid spaces in DMO. ${ }^{11,12,14}$ Chung et al ${ }^{14}$ found an association between visual prognosis and the areas of hyperautofluorescence at the fovea in eyes treated with bevacizumab. The current study investigated for the first time methods for quantifying the signal levels in FAF images and found differences in the FAF signal levels in the parafoveal subfields between eyes with and without DMO. It suggests diagnostic significance of these findings, though we had to consider the limitation of smaller sample number of control eyes. LogMAR VA was also correlated negatively with the mean FAF signals in the individual parafoveal subfields, especially in the nasal subfield. This suggested that quantifying the FAF intensity in the parafoveal areas might be a novel marker of visual impairment in DMO.

The quantification of the FAF signal levels elucidated the clinical relevance of the parafoveal hypoautofluorescence in DMO. It is widely accepted that VA depends on the ability of the cone photoreceptors in the central subfield to perceive light, which is transmitted through secondary or tertiary neurons in the inner retinal layers of the parafoveal areas. ${ }^{21}$ As hypoautofluorescence is related to the increased retinal thickness in the parafoveal subfields, we might hypothesize that hypoautofluorescence reflects the autofluorescence blocked by the oedematous retinal parenchyma, which disturbs signal transduction from the foveal cone photoreceptors. ${ }^{18}$ However, the CSF thickness was also associated negatively with the signal levels of FAF in the parafoveal areas, which might implicate just the statistical correlation.

We considered several possibilities regarding the hypoautofluorescence in the parafoveal subfields, that is, blocked autofluorescence and decreased fluorophores. We often found decreased FAF signals around the autofluorescent cystoid spaces in the parafoveal areas, which seemed to correspond to the cystoid spaces in the OPL on OCT images. ${ }^{14,18,22-24}$ We might speculate that extravasated blood components in the OPL contain 

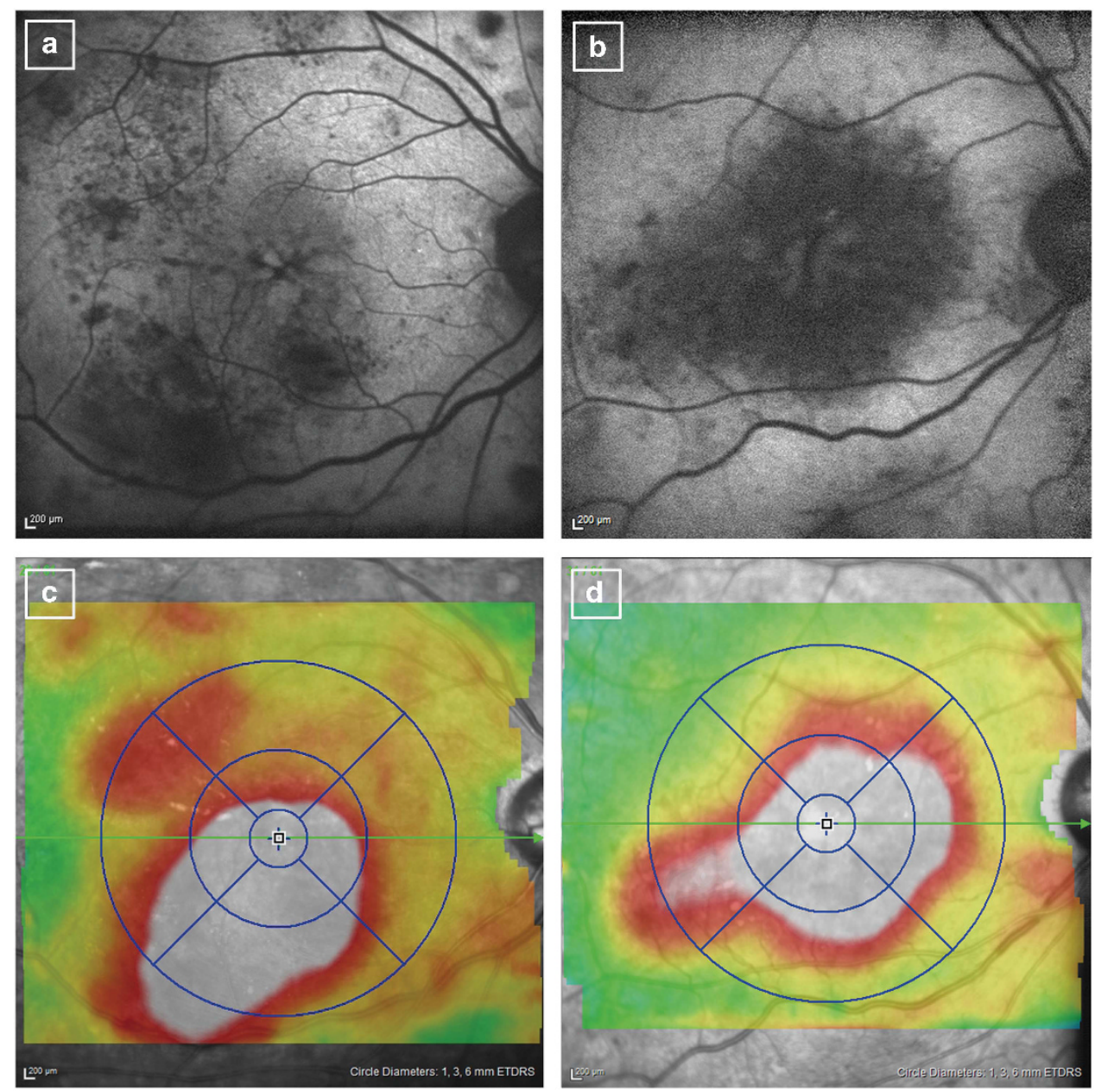

Figure 3 Two representative cases with centre-involved DMO. (a, c) An eye with areas of moderately decreased autofluorescence has macular thickening $(661 \mu \mathrm{m})$ and mild visual impairment (best-corrected decimal visual acuity (BCVA), 1.0). (b, d) An eye with larger areas of macular hypoautofluorescence has greater central subfield thickening $(746 \mu \mathrm{m})$ and severe visual reduction (BCVA, 0.3$)$.

unknown materials that block autofluorescence or decrease the transparency of the resident cells. The optical density of the photoreceptor components might sometimes increase in nonperfused areas, possibly leading to blocked autofluorescence and concomitant hypoautofluorescence. ${ }^{25,26}$ Another explanation might be changes in the origin of the fluorophores. Extravasated blood components from the retinal vasculature might affect the metabolism of the photoreceptor cells and concomitant changes in the fluorophores in these cells or reduce turnover of their outer segments, which could lead to decreased accumulation of lipofuscin. ${ }^{27}$ Because the RPE functions are disrupted by diabetes, another possibility is that phagocytosis of the photoreceptor outer segments might decrease with concomitant lower amounts of fluorophore debris, including lipofuscin. ${ }^{28,29}$

We did not find a relation between the parafoveal FAF signals and foveal SRD. SRD often extends to the parafovea in eyes with DMO and might affect the autofluorescence levels. In eyes with central serous chorioretinopathy, subretinal fluid might block the autofluorescence signals from the RPE or prevent RPE cells from phagocytosing the photoreceptor outer segments. ${ }^{30}$ However, the edge of the hypoautofluorescence areas did not definitively correspond to the area of the SRD in most eyes with $\mathrm{DMO}$, suggesting at least some differences in their pathogenesis.

The levels of the FAF signals in the central subfield were related to the presence of foveal cystoid spaces, which might agree with studies that have described hyperautofluorescence in the areas corresponding to foveal cystoid spaces. ${ }^{11,12,14,31}$ The areas of autofluorescent cystoid spaces at the fovea were reported to be related to visual impairment, retinal thickness, and photoreceptor damage. ${ }^{14}$ Vujosevic et al ${ }^{12}$ reported that eyes with the multiple-spot type of autofluorescent cystoid spaces had poorer VA than those with the 
single-spot type. Compared with those publications, we did not ascertain the diagnostic significance or clinical relevance of the mean autofluorescence levels in the central subfield. We speculated that focal hyperautofluorescence in foveal cystoid spaces might counteract to diffuse hypoautofluorescence due to a few possible mechanisms. ${ }^{32}$ This suggested a limitation in the study methods. Further studies should elucidate how foveal cystoid spaces were evaluated objectively on FAF images. In addition, retinal vasculature, hemorrhages, and hard exudates blocked autofluorescence signals in the parafoveal subfields, which might reduce the mean FAF intensity to a lesser extent.

A recent study reported that several factors affect the autofluorescence levels in healthy subjects: patients' background, that is, age, race, gender, and smoking, and the methods of image acquisition, that is, focus, central alignment, and aperture. ${ }^{33}$ In addition, the cornea or crystalline lens might emit autofluorescent signals in patients with diabetes. ${ }^{16,17}$ This prompted us to investigate the relative autofluorescence levels in the macular subfields compared with the signals outside the ETDRS grid, although a recent publication described a method for quantifying FAF signals using a scanning laser ophthalmoscope equipped with an internal autofluorescent reference. ${ }^{33}$ The advantage of the current method is that the FAF signal levels were adjusted by an internal control within individual images, and the relative autofluorescence levels were, to some extent, independent of the autofluorescence of the optical media. Indeed, we did not find the differences of the relative FAF intensities between phakic and pseudophakic eyes with DMO (data not shown). However, we could not evaluate the absolute value of the FAF levels, compared with the methods reported by Greenberg et al. ${ }^{33}$

Despite the clinical relevance of FAF in the current study, we consider OCT to be the golden standard for diagnosing and treating DMO., ${ }^{74}$ OCT provides qualitative and quantitative parameters of importance regarding $\mathrm{DMO}$, although it takes longer to obtain higherquality SD-OCT images. ${ }^{35-38} \mathrm{FA}$, an invasive modality, shows vascular hyperpermeability and nonperfusion areas. Hypoautofluorescence might correspond to oedematous changes or intraretinal lesions as discussed previously, although FAF does not differentiate these lesions. As FAF imaging is noninvasive and available within several seconds, clinicians might use this modality to screen for DMO. Colour fundus photography is also noninvasive although it has less potential use for detecting DMO.

In the current study, we showed for the first time the clinical relevance of quantified FAF in DMO, compared with the OCT findings, and the potential utility of FAF for screening DMO.

\section{Summary}

\section{What was known before}

- Short-wavelength autofluorescence has potential utility to detect foveal cystoid spaces in diabetic macular oedema.

- Qualitative findings on short-wavelength autofluorescence are associated with visual prognosis after intravitreal bevacizumab injection for diabetic macular oedema.

- Mosaic pattern of near-infrared autofluorescence is associated with macular thickening and photoreceptor damage in diabetic macular oedema.

What this study adds

- Patients with diabetic macular oedema have lower signal levels of short-wavelength autofluorescence in the parafovea than healthy persons.

- LogMAR VA and macular thickness are negatively correlated with the signal levels of short-wavelength autofluorescence in the parafovea, compared with those at the fovea in diabetic macular oedema.

- The signal levels of short-wavelength autofluorescence in the parafovea were not related to foveal pathomorphologies in diabetic macular oedema.

\section{Conflict of interest}

The authors declare no conflict of interest.

\section{References}

1 Klein R, Klein BE, Moss SE, Cruickshanks KJ. The Wisconsin Epidemiologic Study of Diabetic Retinopathy. XV. The longterm incidence of macular edema. Ophthalmology 1995; 102: 7-16.

2 Gardner TW, Antonetti DA, Barber AJ, LaNoue KF, Levison SW. Diabetic retinopathy: more than meets the eye. Surv Ophthalmol 2002; 47(Suppl 2): S253-S262.

3 Antonetti DA, Klein R, Gardner TW. Diabetic retinopathy. N Engl J Med 2012; 366: 1227-1239.

4 Mohamed Q, Gillies MC, Wong TY. Management of diabetic retinopathy: a systematic review. JAMA 2007; 298: 902-916.

5 Cunningham Jr. ET, Adamis AP, Altaweel M, Aiello LP, Bressler NM, D'Amico DJ et al. A phase II randomized double-masked trial of pegaptanib, an anti-vascular endothelial growth factor aptamer, for diabetic macular edema. Ophthalmology 2005; 112: 1747-1757.

6 Bolz M, Schmidt-Erfurth U, Deak G, Mylonas G, Kriechbaum K, Scholda C. Optical coherence tomographic hyperreflective foci: a morphologic sign of lipid extravasation in diabetic macular edema. Ophthalmology 2009; 116: 914-920.

7 Browning DJ, Glassman AR, Aiello LP, Beck RW, Brown DM, Fong DS et al. Relationship between optical coherence tomography-measured central retinal thickness and visual acuity in diabetic macular edema. Ophthalmology 2007; 114 525-536.

8 Delori FC, Dorey CK, Staurenghi G, Arend O, Goger DG, Weiter JJ. In vivo fluorescence of the ocular fundus exhibits retinal pigment epithelium lipofuscin characteristics. Invest Ophthalmol Vis Sci 1995; 36: 718-729. 
9 Holz FG, Bellman C, Staudt S, Schutt F, Volcker HE. Fundus autofluorescence and development of geographic atrophy in age-related macular degeneration. Invest Ophthalmol Vis Sci 2001; 42: 1051-1056.

10 Murakami T, Akimoto M, Ooto S, Suzuki T, Ikeda H, Kawagoe $\mathrm{N}$ et al. Association between abnormal autofluorescence and photoreceptor disorganization in retinitis pigmentosa. Am J Ophthalmol 2008; 145: 687-694.

11 Bessho K, Gomi F, Harino S, Sawa M, Sayanagi K, Tsujikawa $\mathrm{M}$ et al. Macular autofluorescence in eyes with cystoid macula edema, detected with $488 \mathrm{~nm}$-excitation but not with 580 nm-excitation. Graefes Arch Clin Exp Ophthalmol 2009; 247: 729-734.

12 Vujosevic S, Casciano M, Pilotto E, Boccassini B, Varano M, Midena E. Diabetic macular edema: fundus autofluorescence and functional correlations. Invest Ophthalmol Vis Sci 2011; 52: 442-448.

13 Waldstein SM, Hickey D, Mahmud I, Kiire CA, Charbel Issa $\mathrm{P}$, Chong NV. Two-wavelength fundus autofluorescence and macular pigment optical density imaging in diabetic macular oedema. Eye (Lond) 2012; 26: 1078-1085.

14 Chung H, Park B, Shin HJ, Kim HC. Correlation of fundus autofluorescence with spectral-domain optical coherence tomography and vision in diabetic macular edema. Ophthalmology 2012; 119: 1056-1065.

15 Muqit MM, Gray JC, Marcellino GR, Henson DB, Young LB, Patton $\mathrm{N}$ et al. Barely visible 10-millisecond pascal laser photocoagulation for diabetic macular edema: observations of clinical effect and burn localization. Am J Ophthalmol 2010; 149: 979-986 e972.

16 Kessel L, Moldow B, van Best JA, Sander B. Corneal autofluorescence in relation to permeability of the bloodaqueous barrier in diabetic patients with clinically significant macular edema and in an age-matched control group. Curr Eye Res 2003; 26: 307-312.

17 Helve J, Nieminen $\mathrm{H}$. Autofluorescence of the human diabetic lens in vivo. Am J Ophthalmol 1976; 81: 493-494.

18 Murakami T, Nishijima K, Sakamoto A, Ota M, Horii T, Yoshimura N. Association of pathomorphology, photoreceptor status, and retinal thickness with visual acuity in diabetic retinopathy. Am J Ophthalmol 2011; 151: 310-317.

19 Otani T, Kishi S, Maruyama Y. Patterns of diabetic macular edema with optical coherence tomography. Am J Ophthalmol 1999; 127: 688-693.

20 Murakami T, Nishijima K, Sakamoto A, Ota M, Horii T, Yoshimura N. Foveal cystoid spaces are associated with enlarged foveal avascular zone and microaneurysms in diabetic macular edema. Ophthalmology 2011; 118: 359-367.

21 Provis JM, Diaz CM, Dreher B. Ontogeny of the primate fovea: a central issue in retinal development. Prog Neurobiol 1998; 54: 549-580.

22 Tso MO. Pathology of cystoid macular edema. Ophthalmology 1982; 89: 902-915.

23 Otani T, Kishi S. Correlation between optical coherence tomography and fluorescein angiography findings in diabetic macular edema. Ophthalmology 2007; 114: 104-107.
24 Bolz M, Ritter M, Schneider M, Simader C, Scholda C, Schmidt-Erfurth U. A systematic correlation of angiography and high-resolution optical coherence tomography in diabetic macular edema. Ophthalmology 2009; 116: 66-72.

25 Unoki N, Nishijima K, Sakamoto A, Kita M, Watanabe D, Hangai $\mathrm{M}$ et al. Retinal sensitivity loss and structural disturbance in areas of capillary nonperfusion of eyes with diabetic retinopathy. Am J Ophthalmol 2007; 144: 755-760.

26 Bek T. Transretinal histopathological changes in capillaryfree areas of diabetic retinopathy. Acta Ophthalmol (Copenh) 1994; 72: 409-415.

27 Du Y, Veenstra A, Palczewski K, Kern TS. Photoreceptor cells are major contributors to diabetes-induced oxidative stress and local inflammation in the retina. Proc Natl Acad Sci USA 2013; 110: 16586-16591.

28 Simo R, Villarroel M, Corraliza L, Hernandez C, GarciaRamirez M. The retinal pigment epithelium: something more than a constituent of the blood-retinal barrier-implications for the pathogenesis of diabetic retinopathy. J Biomed Biotechnol 2010; 2010: 190724.

29 Strauss $\mathrm{O}$. The retinal pigment epithelium in visual function. Physiol Rev 2005; 85: 845-881.

30 Spaide RF, Klancnik Jr JM . Fundus autofluorescence and central serous chorioretinopathy. Ophthalmology 2005; 112: 825-833.

31 Pece A, Isola V, Holz F, Milani P, Brancato R. Autofluorescence imaging of cystoid macular edema in diabetic retinopathy. Ophthalmologica 2010; 224: 230-235.

32 Reznicek L, Dabov S, Haritoglou C, Kampik A, Kernt M, Neubauer AS. Green-light fundus autofluorescence in diabetic macular edema. Int J Ophthalmol 2013; 6: 75-80.

33 Greenberg JP, Duncker T, Woods RL, Smith RT, Sparrow JR, Delori FC. Quantitative fundus autofluorescence in healthy eyes. Invest Ophthalmol Vis Sci 2013; 54: 5684-5693.

34 Danis RP, Glassman AR, Aiello LP, Antoszyk AN, Beck RW, Browning DJ et al. Diurnal variation in retinal thickening measurement by optical coherence tomography in centerinvolved diabetic macular edema. Arch Ophthalmol 2006; 124: 1701-1707.

35 Murakami T, Nishijima K, Akagi T, Uji A, Horii T, Ueda-Arakawa $\mathrm{N}$ et al. Optical coherence tomographic reflectivity of photoreceptors beneath cystoid spaces in diabetic macular edema. Invest Ophthalmol Vis Sci 2012; 53: 1506-1511.

36 Maheshwary AS, Oster SF, Yuson RM, Cheng L, Mojana F, Freeman WR. The association between percent disruption of the photoreceptor inner segment-outer segment junction and visual acuity in diabetic macular edema. Am J Ophthalmol 2010; 150: 63-67 e61.

37 Alasil T, Keane PA, Updike JF, Dustin L, Ouyang Y, Walsh AC et al. Relationship between optical coherence tomography retinal parameters and visual acuity in diabetic macular edema. Ophthalmology 2010; 117: 2379-2386.

38 Otani T, Yamaguchi Y, Kishi S. Correlation between visual acuity and foveal microstructural changes in diabetic macular edema. Retina 2010; 30: 774-780. 